\title{
Laser Ultrasound Measurement of Diaphragm Thickness, Young's Modulus and Poisson's Ratio in a MEMS Device.
}

\author{
Campbell McKee, Brian Culshaw, and Richard Leach
}

\begin{abstract}
Laser-generated Lamb waves, coupled with a large bandwidth Michelson interferometer, have been demonstrated to accurately measure the thickness of a MEMS pressure sensor diaphragm in the [110] direction of a silicon wafer. Using the reassigned Gabor time-frequency method to produce group velocity dispersion curves, the technique facilitates the measurement of thickness, Young's modulus and Poisson's ratio from just one non-contact measurement. In this investigation, thickness was determined to be $35.01 \mu \mathrm{m} \pm 0.18 \mu \mathrm{m}$. For comparison, the thickness was measured using an independent optical technique; obtaining a value of $34.60 \mu \mathrm{m} \pm 0.27 \mu \mathrm{m}$. Values for Young's modulus and Poisson's ratio were also determined to be $163 \mathrm{GPa} \pm 11.7 \mathrm{GPa}$ and 0.351 respectively and these are in good agreement with values found in the literature.
\end{abstract}

Index Terms-Elastic modulus, Elasticity, Laser ultrasonics, Microelectromechanical systems (MEMS), Poisson's ratio, Silicon, Young's modulus,

\section{INTRODUCTION}

$\mathrm{M}$ ICROELECTROMECHANICAL systems (MEMS) have dimensions that fall broadly within the $1 \mu \mathrm{m}$ to 1 $\mathrm{mm}$ range and combine electrical and mechanical components. The MEMS pressure sensor is one such system. With the continued growth of the MEMS industry, there is an everincreasing need to measure the dimensions and material properties of these structures - both on the silicon wafers and the final pressure sensor membrane, not only to ensure reliability but also to improve future designs. One concern is that the material properties at the microscale can vary significantly compared to those of the bulk material [1]. The preliminary results reported here show close correlation with bulk values for this particular device process.

The main applications of MEMS pressure sensors in the

This research was funded by an EPSRC grant (EP/E053319/1) and complimented by a CASE award from the National Physical Laboratory (NPL).

C. McKee was with the Centre for Microsystems and Photonics, University of Strathclyde, Glasgow G1 1XW. He is now with James Fisher Testing Services, Bristol, BS5 0SP. (e-mail: c.mkee@ strainstall.com).

B. Culshaw is professor emeritus at the Centre for Microsystems and Photonics, University of Strathclyde, Glasgow G1 1XW. (e-mail: brian.culshaw@strath.ac.uk).

R. Leach was with the National Physical Laboratory (NPL), Teddington. $\mathrm{He}$ is now with the Manufacturing Metrology Team, Faculty of Engineering, University of Nottingham, NG7 2RD. (e-mail: richard.Leach@nottingham.ac.uk). automotive industry are manifold air pressure sensors and common fuel rail pressure sensors for engine management systems [2]. In the medical industry, MEMS pressure sensors are used in applications such as disposable devices for catheters employed in surgical operations [3], and for pressure and differential flow monitoring in continuous positive airway pressure machines for treating sleep apnea [4]. The construction industries use MEMS pressure sensors to measure airflow [5], while the aerospace industry uses MEMS pressure sensors to monitor engines, flaps and other functions, and to provide precision altitude air pressure measurement [6].

This work was conducted on the diaphragms of pressure sensors etched into silicon wafers. This is the only method, as far as we know that can deduce the described mechanical properties from a single measurement. Others, have employed laser generated surface acoustic waves using commercial instrumentation [27] to obtain thin film thickness on a large substrate and additionally used a nanoindentation technique to determine mechanical properties. However, this is a destructive technique [28]. A full review of applications and current characterization techniques is given in [22]. Due to the potential speed of the test procedure, it could be possible to automate this as a test mechanism for use during the manufacturing process.

\section{LAMB WAVES AND TIME FREQUENCY ANALYSIS}

\section{A. Lamb Waves}

Lamb waves are ultrasonic guided waves which occur in thin plates, where the planar dimensions are much greater than the thickness and where the wavelength is of the order of the thickness [7]. They are formed by the interference of multiple reflections and mode conversion of longitudinal and transverse waves at the surfaces of the plate. These waves propagate as so called symmetric and antisymmetric modes, so called because of the relative displacement patterns on the two plate surfaces (Fig. $1 \&$ Fig. 2)

These modes are superpositions of longitudinal and shear vertical waves and each wave type can propagate independently of the other. Fig. 1 and Fig. 2 show a representation of the particle motion in the material. The symmetric and anti-symmetric modes are normally abbreviated to $S_{i}$ and $A_{i}(i=0,1, \ldots)$ respectively. The symmetric modes normally have a dominant in-plane motion 
compared to the dominant out-of-plane motion of the antisymmetric modes. Each of these wave modes are governed by their own equation [9].

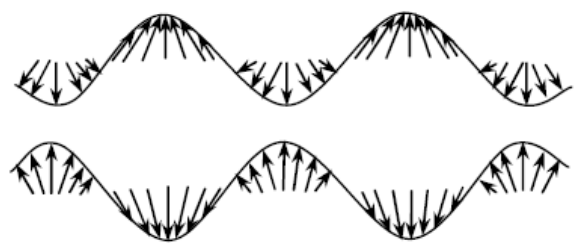

Fig. 1. Particle motion of the symmetric Lamb wave mode [8].

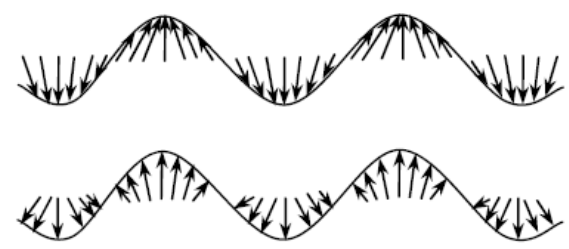

Fig. 2. Particle motion of the antisymmetric Lamb wave mode [8].

These equations are the well know Rayleigh-Lamb dispersion equations and are given below. Symmetric modes are defined as

$$
\frac{\tan (q h)}{\tan (p h)}=-\frac{4 k^{2} q p}{\left(k^{2}-q^{2}\right)^{2}}
$$

and antisymmetric modes are defined as

$$
\frac{\tan (q h)}{\tan (p h)}=-\frac{\left(k^{2}-q^{2}\right)^{2}}{4 k^{2} q p}
$$

where, $h=d / 2, d$ is the plate thickness, $k$ is the wavenumber, $\omega$ is the angular frequency and $p$ and $q$ are given by

$$
p^{2}=\frac{\omega^{2}}{C_{L}^{2}}-k^{2} \quad q^{2}=\frac{\omega^{2}}{C_{T}^{2}}-k^{2}
$$

where $C_{L}$ and $C_{T}$ are the longitudinal and transverse wave velocities respectively. The velocities are a function of the wave's frequency and the plate thickness, making Lamb waves dispersive.

The longitudinal and transverse wave velocities can be related to the material elastic properties by [9]

$$
\begin{gathered}
C_{L}=\sqrt{\frac{\lambda+2 \mu}{\rho}}=\sqrt{\frac{E}{\rho} \frac{(1-v)}{(1+v)(1-2 v)}} \\
C_{T}=\sqrt{\frac{\mu}{\rho}}=\sqrt{\frac{E}{\rho} \frac{1}{2(1+v)}}
\end{gathered}
$$

where $\lambda$ and $\mu$ are the Lamé constants, and can be expressed as

$$
\lambda=\frac{v E}{(1-2 v)(1+v)} \quad \mu=\frac{E}{2(1+v)^{2}}
$$

$E$ is Young's modulus, $\rho$ is the material density and $v$ is Poisson's ratio. If $C_{L}, C_{T}$ and $\rho$ are known, Poisson's ratio and Young's modulus are given by: [9]

$$
\begin{gathered}
v=\frac{1-2\left(\frac{C_{T}}{C_{L}}\right)^{2}}{2-2\left(\frac{C_{T}}{C_{L}}\right)^{2}} \\
E=2 \rho C_{T}^{2}(1+v) .
\end{gathered}
$$

The Rayleigh-Lamb wave equations are used to obtain velocity dispersion curves. These dispersion curves can either be wavenumber, phase velocity or group velocity dispersion curves, and the choice of which to use ultimately depends on the measurement type and experimental set up. Lamb waves, can travel with both a phase and group velocity. The phase velocity is the velocity at which an individual component of a wave propagates and is given as $c_{p}=\omega / k$, whereas the group velocity is the velocity of the guided wave packet and is given as $c_{g}=\delta \omega / \delta k$. The group velocity defines the speed and direction of the flow of energy transmitted by the wave. Fig. 3 shows an example of the group velocity dispersion curve calculated for $50 \mu \mathrm{m}$ [110] silicon. These dispersion curves were produced using a MATLAB programme developed within the University of Strathclyde Centre for Ultrasonic Engineering [10]. It is clear that as the frequency increases, so does the number of modes, and so theoretically, an infinite number of modes can exist in a plate, all of which are dispersive at some point. However, below a certain cut-off threshold, only the two fundamental modes, $S_{0}$ and $A_{0}$, can propagate. . These curves vary slightly in detail for different crystal directions due to different effective physical constants.

The non-dispersive, low frequency component of the $S_{0}$ mode is known as the plate wave velocity and is given by

$$
\lim _{f d \rightarrow 0} c_{s}=\sqrt{\frac{E}{\rho\left(1-v^{2}\right)}}
$$

where $c_{s}$ is the symmetric Lamb wave velocity or plate wave velocity. As the frequency increases, the fundamental symmetric and antisymmetric modes converge to the Rayleigh wave velocity, $C_{R}$ [9]. The dispersion equations and the resulting dispersion curves are used to describe the relationship between frequency, sample thickness and phase or group velocity. Fig. 3 with (1) and (2) shows that:

- The wave modes are a function of the product of the frequency and sample thickness.

- The lower order modes $\left(A_{0}\right.$ and $\left.S_{0}\right)$ exist for all frequencies and the higher order modes $\left(A_{i}\right.$ and $S_{i}(i=1$, $2 \ldots))$ appear with increasing frequency. 
- There are regions of low dispersion for the lower order modes ( $\approx 50 \mathrm{MHz}$ for the case analyzed in Fig. 3), before the higher order modes begin to appear.

One commonly used method for obtaining Lamb wave phase velocity dispersion curves is by using the two dimensional Fourier transform (2D-FT) as described in [26]. This signal processing method requires hundreds of measurements and is only applicable to relatively large structures. A much simpler solution would be to use a signal processing technique that only requires one measurement.

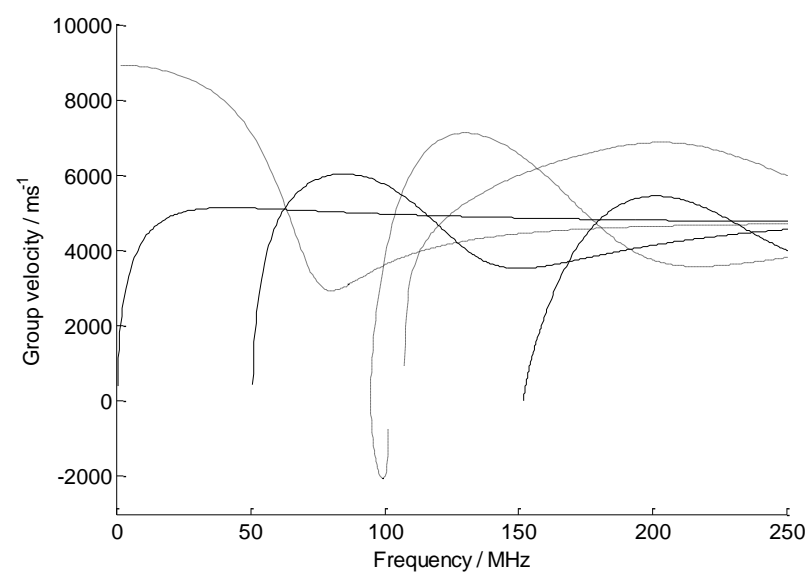

Fig. 3. Lamb wave group velocity dispersion curves for a $50 \mu \mathrm{m}$ silicon wafer in the [110] direction. Symmetric modes are shown as dashed lines. Anti-symmetric modes are shown as solid lines

\section{B. Time-frequency analysis of Lamb waves}

As first stated by Gabor [11], it is possible to map a one dimensional time domain signal into a two dimensional time and frequency signal, which represents the variation of spectral energy over time [12]. Lamb waves are an example of a non-stationary multimode signal making it suitable for time-frequency analysis. Time-frequency analysis is able to resolve individual Lamb modes, leading to a group velocity representation [13].

\section{Short time Fourier Transform}

The short time Fourier transform (STFT) contains a windowing function that, when applied to a signal, breaks the signal into segments, where a Fourier transform is performed. This is mathematically defined as

$$
\operatorname{STFT}(x ; \omega, t)=\int_{-\infty}^{\infty} x(\tau) h(\tau-t) e^{-i 2 \pi \omega \tau} d \tau
$$

The window, $h(\tau-t)$ suppresses the signal around the analysis time point, $\tau=t$, and the STFT gives a local spectrum of the signal $x(\tau)$ around $t$. The output of the STFT is the spectrogram and is the energy density spectrum of the STFT. This is given as

$$
E(\omega, t) \propto|S(\omega, t)|^{2} .
$$

The STFT, as with all time-frequency representations, suffers from the Heisenberg-Gabor uncertainty principle. This states that it is impossible to simultaneously obtain good resolution in time and frequency. The resolutions in time and frequency are related and limited by the inequality:

$$
\sigma_{t}^{2} \sigma_{\omega}^{2} \geq \frac{1}{4}
$$

where $\sigma_{t}$ is the standard deviation for time and $\sigma_{\omega}$ is the standard deviation for frequency. The resolution in time and frequency of the spectrogram is dictated completely by the window size and type used. A narrow window will give good time resolution and poor frequency resolution, while a wide window gives good frequency resolution and poor time resolution.

\section{The reassignment method}

The reassignment method [14] provides a method for "cleaning up" the spectrogram. In the reassignment method, the energy of the signal is moved from its original location $(t, \omega)$ to a new location $(\hat{t}, \widehat{\omega})$, reducing the spread of the spectrogram and improving its resolution by concentrating its energy at a "center of gravity" [14]. It was shown in [14] that the reassigned coordinates $\hat{t}$ and $\widehat{\omega}$ for a spectrogram are

$$
\hat{t}=t-\Re\left(\frac{S_{\mathcal{T} h}(x, t, \omega) \cdot \overline{S_{h}(x, t, \omega)}}{\left|S_{h}(x, t, \omega)\right|^{2}}\right)
$$

and

$$
\widehat{\omega}=\omega-\mathfrak{J}\left(\frac{S_{\mathcal{D} h}(x, t, \omega) \cdot \overline{S_{h}(x, t, \omega)}}{\left|S_{h}(x, t, \omega)\right|^{2}}\right)
$$

where $S_{h}(x, t, \omega)$ is the standard STFT of the signal $x$ using window function $h(t)$ and $S_{T h}(x, t, \omega)$ is the STFT using a timeramped version of the window, $t \cdot h(t)$ and $S_{D h}(x, t, \omega)$ is the STFT using the first derivative of the window function, $\frac{d h(t)}{d t}$ [14].

The reassignment method can be considered a two-step process:

- Smoothing - the purpose is to smooth oscillatory interference but has the disadvantage of smearing localised components.

- $\quad$ Squeezing - the purpose is to refocus the contributions that survived the smoothing.

\section{Generating Guided Elastic WAVES IN A MEMS PRESSURE SENSOR}

The Lamb waves were excited in the diaphragm of a MEMS pressure sensor using a broadband laser source. Fig. 4 shows the pressure sensor diaphragms etched into a (100) ntype silicon wafer with stiffening boss [15]. Also visible is a blank test plate (circled) used for the measurements. The diaphragms are approximately $2.5 \mathrm{~mm}$ square. The sample was mounted vertically in between the generation source laser and 
a Michelson interferometer, and was held in place using magnetic strips. The generation source used was a Teem Photonics Powerchip PB Nano UV laser (PowerChip-PNVB25010-130) with wavelength $355 \mathrm{~nm}$, pulse energy of $28 \mu \mathrm{J}$, pulse width of $400 \mathrm{ps}$ and repetition rate of $10 \mathrm{~Hz}$. The laser was mounted on a Newport motion controller translation stage (MM4005) which was used to adjust the laser in the y-axis, to adjust the source-detector distance and in the $\mathrm{x}$-axis, to adjust the focusing of the laser beam. The beam was focused onto the sample using a UV cylindrical lens. The cylindrical lens focuses the beam to a line, reducing the energy density and avoiding ablation. The detection scheme was a custom built Michelson interferometer with $200 \mathrm{MHz}$ bandwidth [22]. The optimal focus was obtained by firing the laser and finely adjusting the translation stage until the signal with the maximum signal to noise ratio was observed on an oscilloscope. The oscilloscope used was an Agilent Infininium 54832D MSO and the signals were averaged 4096 times with each measurement. The experimental set up is described in detail in [22].

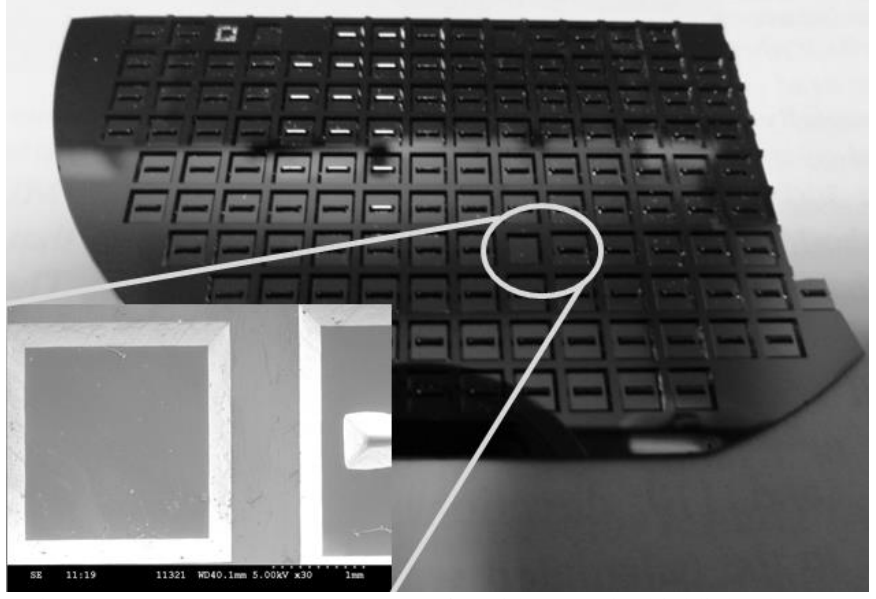

Fig. 4. MEMS pressure sensor on wafer with test point highlighted. Insert shows SEM image of test point

\section{CHARACTERISATION OF THE MEMS PRESSURE SENSOR - RESULTS AND DISCUSSION}

Fig. 5 shows the measured signal while Fig. 6 shows the same data filtered using a Chebyshev Type II digital bandpass filter between $5 \mathrm{MHz}$ and $250 \mathrm{MHz}$. The Chebyshev filter is more suitable when the frequency content of a signal is more important. Below $5 \mathrm{MHz}$ the ultrasonic wavelength well exceeds the diaphragm dimensions The signal is off large amplitude and long wavelength and any delay measurements are very strongly influenced by the surrounding silicon substrate which are consequently extremely difficult to interpret. Indeed Fig. 6 does have evident structure below the nominal $5 \mathrm{MHz}$ cut off indicating strong interference.

To obtain the group velocity dispersion curve, the reassigned Gabor transform was applied to the data using the MATLAB Time-Frequency Toolbox [16].
The Gabor transform is similar to the STFT with the exception that the Gabor transform uses a Gaussian window with much improved time and frequency resolution compared to the STFT. This resulted in the time-frequency representations of Fig. 7. For the Gabor transform, the window length is given as $\mathrm{N} / 4$, where $\mathrm{N}$ is the length of the analysed signal. In this case the window length is $256 \mathrm{~ns}$.

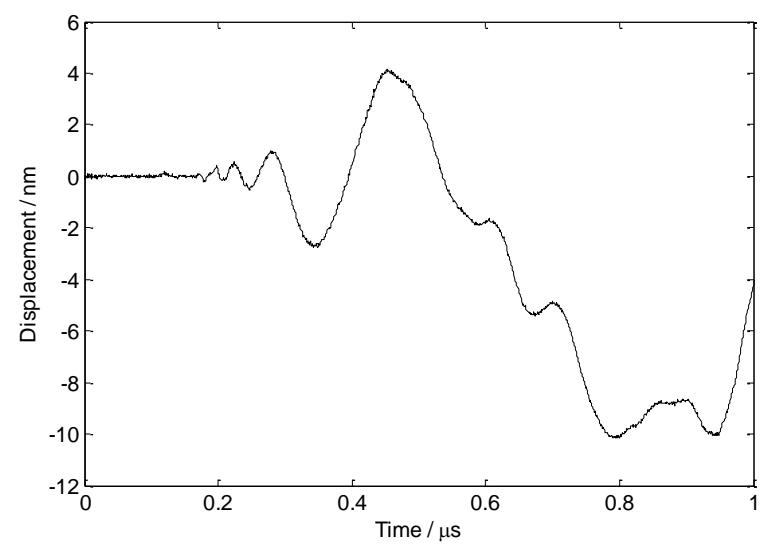

Fig. 5. Measured Lamb wave generated in MEMS pressure sensor.

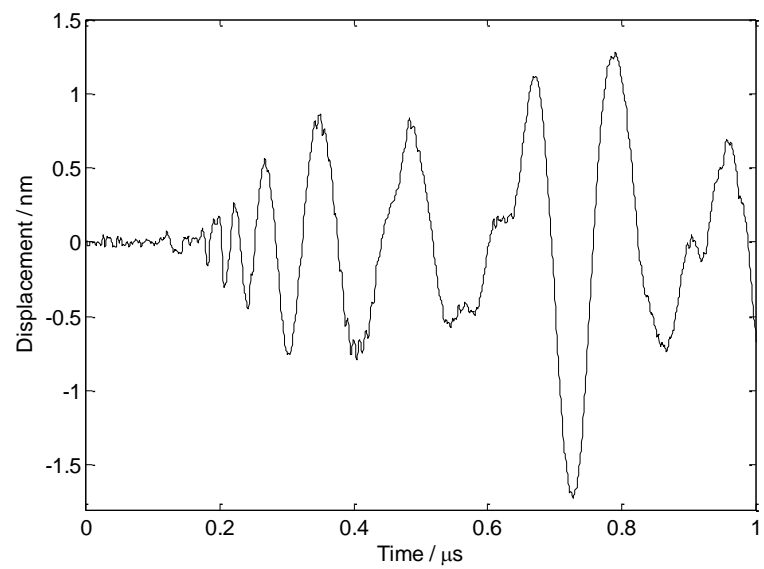

Fig. 6. Measured data after bandpass filter between $5 \mathrm{MHz}$ and $250 \mathrm{MHz}$ is applied.

Fig. 7 contains all the information needed to determine the thickness of the membrane along with values for group velocity, plate velocity, Rayleigh velocity, Young's modulus and Poisson's ratio. Three measurements, plate velocity, Rayleigh velocity and $S_{1}$ mode zero group velocity give all the information needed to derive these important parameters.. Note that there is no closed form mathematical expression to relate group velocity to elastic constants, which may be manipulated to obtain elastic constants. Developing such an expression would be highly complex and would present severe challenges for computerised optimisation [17]. Therefore, the practical method for obtaining material properties is by measuring the group velocities of each mode at appropriately selected frequencies and using the equations (3) to (6). To determine the material properties, some assumptions have to be made. It is assumed that the density is $2329 \mathrm{kgm}^{-3}$ and that the transverse wave velocity in the [110] direction is well 
documented and given as $5844 \mathrm{~ms}^{-1}$ [18].

There is every likelihood that the wafer has been doped with another element. It is, however, generally accepted that doping has no effect on material properties including wave velocities, assuming doping levels are $<10^{20} \mathrm{~cm}-3$ [19]. For very heavy doping, the mechanical properties such as Young's modulus will typically decrease by approximately $1 \%$ to $3 \%$ [20]. Finally, the source-detector propagation distance is 1.25 $\mathrm{mm}$.

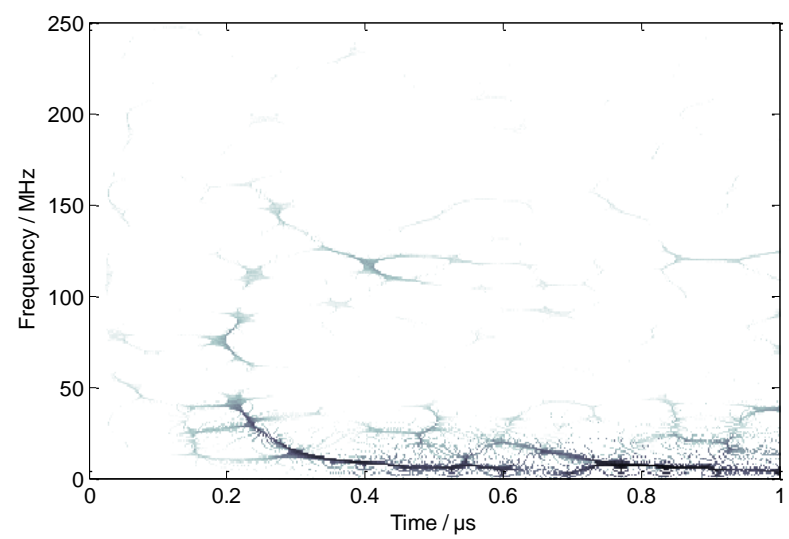

Fig. 7. Reassigned Gabor time-frequency representation on the detected Lamb wave measured in the MEMS pressure sensor membrane.

\section{A. Plate velocity}

An important feature is the non-dispersive component of the $S_{0}$ mode, i.e. the low frequency component, known as the plate velocity, defined in (7). It is clear that changes in both Young's modulus and Poisson's ratio have a large impact on the plate velocity in the group velocity dispersion curve, but thickness has no impact. In an anisotropic material, in a direction of high symmetry, it can be assumed that the plate velocity and group velocity of the low frequency $S_{0}$ mode are equal [17].

Fig. 7 shows the arrival time of the $S_{0}$ mode. Note that there is spurious structure in these results due to some extent to system noise and also due to multipath effects. Consequently we use prior knowledge of the direct source to detector path length to assist in isolating important features in the results.

Fig. 7 also shows a high region of dispersion in the $A_{0}$ mode. Concentrating on the $S_{0}$ mode, the arrival time occurs at $0.14 \mu$ s. Considering a propagation distance of $1.25 \mathrm{~mm}$, this equates to a group velocity of $8930 \mathrm{~ms}^{-1} \pm 320 \mathrm{~ms}^{-1}$. The quoted error is due to the uncertainty in the propagation distance. This is the value for the plate velocity within the membrane. The $A_{0}$ mode, shown in Fig. 7 from approximately $0.2 \mu$ s and under approximately $50 \mathrm{MHz}$ is highly insensitive to variations in mechanical properties and as such can be ignored. This results from the implications of window length (256 ns) and frequency resolution highlighted in (10)

\section{B. Rayleigh velocity}

When the fundamental symmetric and anti-symmetric Lamb wave modes begin to converge, the Lamb waves start to make the transition to a Rayleigh wave. In other words the frequency of the wave has increased and the wavelength decreased to a smaller dimension relative to the plate or membrane thickness.

The time-frequency plot in Fig. 7 shows the point where the $A_{0}$ and $S_{0}$ modes almost meet. Note that is the point where the two fundamental modes converge and the group velocity for both modes becomes the Rayleigh wave velocity.

The two modes can be seen converging in Fig. 8., with the $A_{0}$ mode on the left and the $S_{0}$ mode on the right. Fig. 8. shows a magnified region of Fig. 7 .

Knowing the source-detector separation distance, the Rayleigh wave velocity was calculated to be $5465 \mathrm{~ms}^{-1} \pm$ $145 \mathrm{~ms}^{-1}$.

Fig. 8. highlights a problem with the reassigned timefrequency method. On the left, is some structure believed to be the Rayleigh wave associated with the $A_{0}$ mode. On the right is the $S_{0}$ mode approaching the Rayleigh velocity. In between these two modes, however, is interference caused by two closely-spaced components. With time-frequency representations, there is a trade-off between resolution and localisation (10).If more than one component is seen within a time-frequency smoothing window, a beating effect occurs, causing interference fringes.

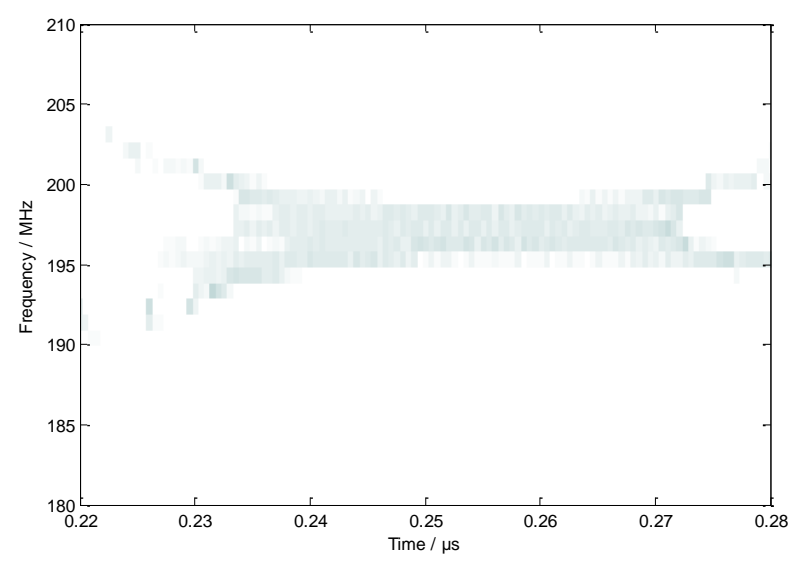

Fig. 8. Onset of Rayleigh wave.

\section{The $S_{1}$ mode and membrane thickness}

One interesting property of Lamb waves is that at specific frequencies, the group velocity tends towards zero, while the phase velocity remains finite. This is illustrated in Fig. 3, where the $S_{I}$ mode crosses the y-axis, i.e. when the $S_{I}$ mode is zero. When the phase velocity becomes infinite, the group velocity is zero, meaning the plate vibrates in longitudinal or shear thickness mode resonance. These resonances become uniformly distributed on the plate surface. The minimum Lamb wave mode where this is observed is within the $S_{1}$ mode, but it can also occur in higher modes. The $S_{1}$ mode has been shown to have a very large quality factor [21] hence at the zero group velocity point there will be a peak in frequency response, making it ideal for bulk acoustic wave velocity and thickness measurements. The maximum peak shown at $\approx 120$ $\mathrm{MHz}$ is the $S_{1}$ resonant mode. Fig. 9 shows other peaks of 
lower amplitudes. These are other elements of the signal evident in Fig. 10

The $S_{1}$ mode (along with other higher order modes) originates at a particular cut-off frequency. At this frequency, $f_{R}$, the plate vibrates in longitudinal or shear thickness mode resonance. For the $S_{1}$ mode, this point is a function of longitudinal velocity, $C_{L}$ and the plate thickness, $h$, thus

$$
f_{R}=\frac{C_{L}}{2 h} .
$$

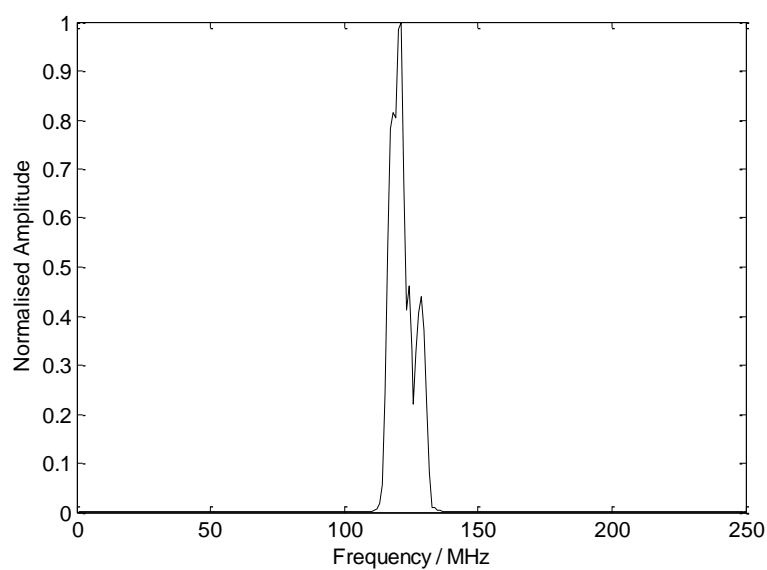

Fig. 9. FFT of measured signal. $\mathrm{S} 1$ mode is visible at $\approx 120 \mathrm{MHz}$.

The origins of the $S_{1}$ zero group velocity resonance are within the behaviour of the dispersion curve. A more detailed explanation of the formation of these modes is given in [21]. Fig. 10 shows that the zero group velocity of the $S_{1}$ mode occurs at a frequency of $120.43 \mathrm{MHz} \pm 0.64 \mathrm{MHz}$, equating to a thickness of $35.01 \mu \mathrm{m} \pm 0.18 \mu \mathrm{m}$ using (13).

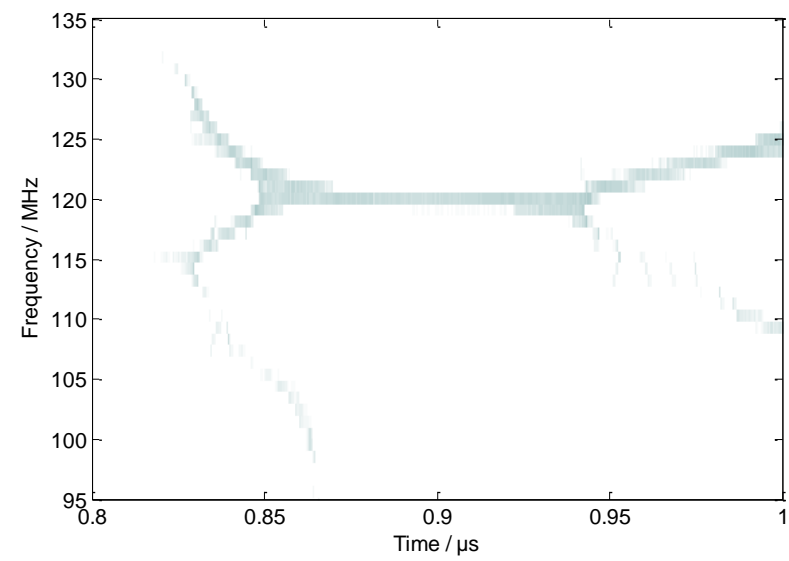

Fig. 10. Measure S1 mode approaching zero group velocity.

The $S_{1}$ mode at zero group velocity is also very sensitive to changes in thickness [21]. The sensitivity to change in thickness $\delta h$ can be given as

$$
\delta h=\frac{h \delta f}{f}
$$

where $\delta f$ and $f$ are the change in frequency shift and frequency respectively. If the resonance peak shifts on the order of $0.1 \mathrm{MHz}$, and with a measured frequency of $120 \mathrm{MHz}$ and a thickness of $35 \mu \mathrm{m}$, changes of thickness of $292 \mathrm{~nm}$ (approximately $0.8 \%$ ) can be observed.

\section{Estimation of Young's modulus and Poisson's ratio in the} [110] direction

The solution to the Rayleigh wave equation is given as [29]

$$
C_{R}=\frac{0.862+1.14 v}{1+v} C_{T}
$$

where $C_{R}$ is the Rayleigh velocity, $v$ is Poisson's ratio and $C_{T}$ is the transverse velocity.

Using (15), a value for Poisson's ratio of 0.351 is obtained. Having calculated a value for the plate velocity and Poisson's ratio, Young's modulus can now be found. Using (7), Young's modulus can be shown to be $163 \mathrm{GPa} \pm 11.7 \mathrm{GPa}$.

\section{E. Experimental and theoretical results}

This final section pulls together the measured timefrequency spectrogram and using the determined material properties, group velocity dispersion curves are given. Here the very low surface displacements become more susceptible to noise and interference. There is also some overlap with the $\mathrm{S}_{1}$ in this region. At low frequencies $(<\approx 10 \mathrm{MHz})$ the multipath effects produce many delayed signals, apparently strong due to the higher displacements at lower frequencies, which are evident in the lower section of the graph. The experimentally derived group velocity dispersion curves are compared to those that has been theoretically calculated in Fig. 11 for fundamental modes and higher modes, showing good agreement between the theoretical and measured points taking into account multipath effects and interference caused by the time frequency windowing. The dispersion curves were obtained using the MATLAB "ginput" function which relies on human input. Future work could include automating this process.

In this plot, the mostly dominant source of error is the distribution of the points during the reassignment process caused by neighbouring modes. In the group velocity dispersion curve, these appear isolated. However, upon comparing the group velocity dispersion curve with the timefrequency dispersion of Fig. 7, it is clear that there are a considerable number of locations where this interference can occur. As discussed above, this is unfortunately unavoidable. 


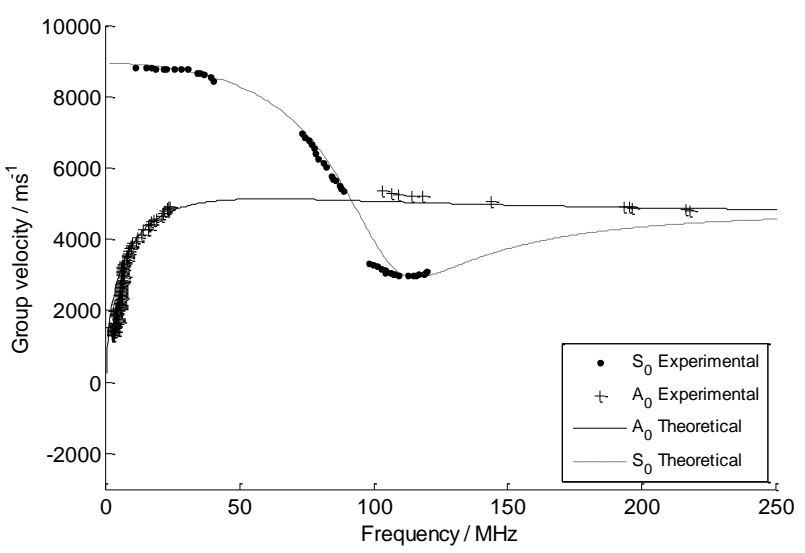

(a)

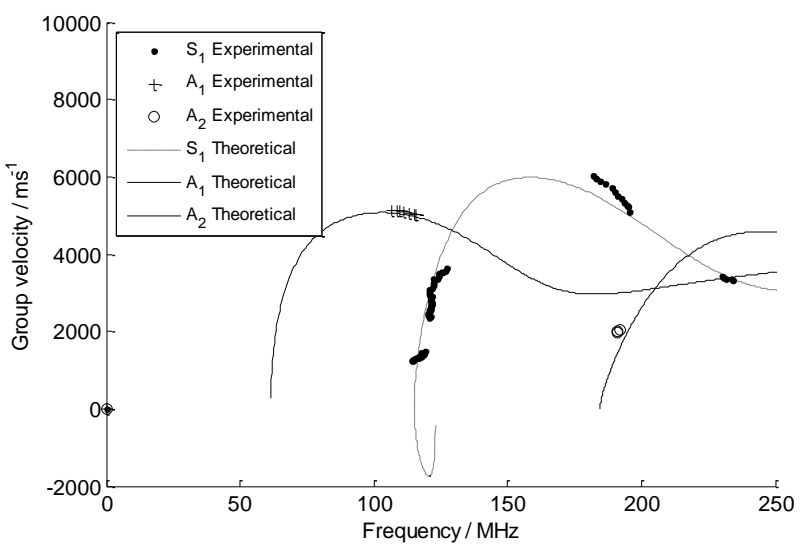

(b)

Fig. 11. (a) Measured and theoretical fundamental (A0 \& S0) group velocity dispersion curves for MEMS pressure sensor membrane. (b) Measured and theoretical higher $\left(\mathrm{S}_{1}, \mathrm{~A}_{1} \& \mathrm{~A}_{2}\right)$ group velocity dispersion curves for MEMS pressure sensor membrane.

\section{MEMBRANE THICKNESS MEASUREMENT - COMPARISON WITH ALTERNATE OPTICAL TECHNIQUE}

The discussion above shows that by applying timefrequency analysis to a multimode Lamb wave signal in a MEMS pressure sensor membrane, the membrane thickness among other properties can be determined.

Another method suitable for measuring the thickness of an optically transparent material is to use light within a particular wavelength range where the material is partially transparent. In this case, the two surfaces of the material would act as an etalon. If the refractive index of the sample is known, the separation of the surfaces, i.e. the sample thickness can be determined by measuring the interference between directly transmitted and internally reflected light. Interference produces intensity modulation with peaks occurring when the optical path length difference (2nd) between the two paths is an integer number of wavelengths. The difference between the peaks is the free spectral range $\Delta v$ (wavenumber) or $\Delta \lambda$ (wavelength) and these are given by

$$
\Delta v=\frac{c}{2 n d} \quad \Delta \lambda=\frac{\lambda^{2}}{2 n d}
$$

where $c$ is the speed of light in vacuum, $\lambda$ is the wavelength, $n$ is the real part of the complex refractive index and $d$ is the plate thickness.

The laser source was a Santec TSL210 fibre laser with a wavelength range of $1530 \mathrm{~nm}$ to $1610 \mathrm{~nm}$. The light was delivered via an optical fibre and was incident perpendicular to the sample. The transmitted light was focused onto a photodiode using a $\times 10$ microscope objective and the signal was amplified using a Fempto Amplifier (DHPCA-100).

Over the wavelength range produced by the laser, there is a change in refractive index of the sample. This was corrected for using the Sellmeier equation for silicon [23]-[25].

Fig. 12 shows the transmission spectrum for the MEMS pressure sensor. From the measured peaks, the thickness of the MEMS pressure sensor membrane was calculated to be 34.6 $\mu \mathrm{m} \pm 0.27 \mu \mathrm{m}$.

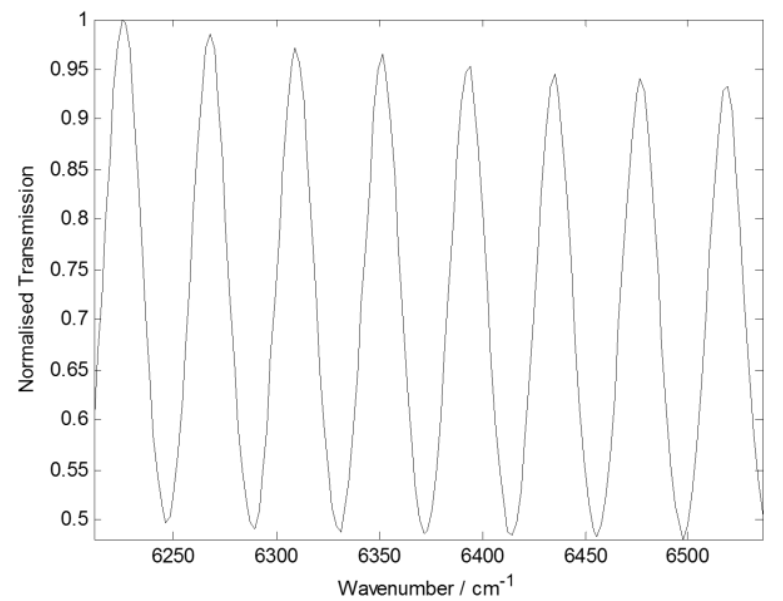

Fig. 12. Infrared transmission spectrum for the MEMS pressure sensor membrane.

\section{CONCLUSIONS}

It has been shown that, even by taking a single measurement and performing time frequency analysis, an accurate value for the membrane thickness of a MEMS pressure sensor can be calculated using the $S_{1}$ Lamb wave mode. This was calculated to be $35.01 \mu \mathrm{m} \pm 0.1 \mu \mathrm{m}$. Using a comparable technique, the membrane thickness was determined to $34.6 \mu \mathrm{m} \pm 0.27 \mu \mathrm{m}$. It is reasonable to conclude that these two results are in good agreement with each other. The test points had a thin metallic film on the laser excitation side. While this enhanced the absorbed energy used to generate the acoustic waves, it had to be removed to allow for light transmission for the comparison test. The small discrepancy could also be due to the fact that both measurements were not taken at the same location, allowing for thickness variations within the membrane.

Values of $163 \mathrm{GPa} \pm 11.7 \mathrm{GPa}$ for Young's modulus and 0.351 for Poisson's in the [110] direction have also been obtained from plate velocity and Rayleigh wave velocity measurements.. Comparing these to values taken from [20], Hopcroft quotes $169 \mathrm{GPa}(3.6 \%$ greater than the value obtained in this work) for Young's modulus and $0.36(2.5 \%$ 
greater that the value obtained in this work) for Poisson's ratio.

\section{ACKNOWLEDGMENT}

C. McKee thanks G. Pierce, G. Thursby, A. Cleary, I Armstrong, I. Veres, G Flockhart, M Lengdon and G Brown. from the University of Strathclyde and N. Jennett from NPL. C. McKee would also like to thank R. Jones from GE Sensing for the MEMS pressure sensor samples and B.Sorazu from the University of Glasgow for advice on signal analysis.

\section{REFERENCES}

[1] A. R. Jha, "Materials for MEMS and Nanotechnology based Sensors and Devices," in MEMS and Nanotechnology based Sensors and Devices for Communications, Medical and Aerospace Applications, CRC Press, 2008.

[2] W. S. Czarnocki and J. P. Schuster, "The Evolution of Automotive Pressure Sensors," Sensors, vol. 16, no. 5, pp. 52-65, 1999.

[3] K. J. Rebello, "Applications of MEMS in surgery," Proceedings of the IEEE, vol. 92, no. 1, pp. 43-55, 2004.

[4] P. K. Kumar, V. K. Nithya and J. A. Vimala, "Micro System with MEMS Sensor for Detecting Sleep Apnea," Journal of Engineering and Applied Sciences, vol. 11, no. 3, pp. 2097-2101, 2016

[5] L. Lofdahl and M. Gad-al-Hak, "MEMS-based pressure and shear stress sensors for turbulent flows," Meas. Sci. \& Technol., vol. 10, no. 8, pp. 665-686, 1999

[6] N. Maluf and K. Williams, An Introduction to Microelectromechanical Systems Engineering, 2nd ed., Artech House, 2004.

[7] J. L. Rose, Ultrasonic Waves in Solid Media, Cambridge University Press, 2004.

[8] Z. Su and L. Ye, "Identification of Damage Using Lamb Waves: From Fundamentals to Applications," in Lecture Notes in Applied and Computational Mechanics, vol. 48, F. Pfeiffer and P. Wriggers, Eds., Springer, 2009, pp. 15 - 53.

[9] W. P. Rogers, "Elastic property measurement using Rayleigh-Lamb waves," Res. Nondestr. Eval, vol. 6, pp. 185 - 208, 1995.

[10] I. Veres, Private Communication, 2010.

[11] D. Gabor, "Theory of communication. Part 1: The analysis of information.," J.IEE, vol. 93, no. 26, 1946.

[12] K. R. Fitz and S. A. Fulop, "A unified theory of time-frequency reassignment," 2009. arXiv:0903.3080v1 [cs.SD]. [Online]. Available: http://arxiv.org/abs/0903.3080.

[13] S. Hurlebaus, M. Niethammer, L. J. Jacobs and C. Valle, "Automated methodology to locate notches with Lamb waves," Acoustics Research Letters Online, vol. 2, no. 4, pp. 97-102, 2001.

[14] F. Auger and P. Flandrin, "Improving the readability of time-frequency and time-scale representations by the reassignment method," IEEE. Trans. Signal Processing, vol. 43, no. 5, pp. 1068-1089, 1995.

[15] P. K. Kinnell, J. King, M. Lester and R. Craddock, "A hollow stiffinging structure for low pressure sensors," Sens. Actuators, A, vol. 160, pp. 35 41,2010

[16] F. Auger, P. Flandrin, P. Goncalves and O. Lemoine, Time-Frequency Toolbox: For use with MATLAB, CNRS \& Rice University, 1996.

[17] A. G. Every and W. Sachse, "Determination of the elastic constants of anisotropic solids from acoustic-wave group-velocity measurements," Phys. Rev. B, vol. 42, no. 13, pp. 8196-8205, 1990.

[18] H. J. McSkimin and P. Andreatch, "Elastic moduli of silicon vs hydrostatic pressure at $25.0^{\circ} \mathrm{C}$ and $-195.8^{\circ} \mathrm{C}$," J. Appl. Phys., vol. 35, no. 7, pp. 2161-2165, 1964.

[19] W. C. Crone, "A Brief Introduction to MEMS and NEMS," in Springer Handbook of Experimental Solid Mechanics, W. N. Sharpe, Ed., 2008.

[20] M. A. Hopcroft, W. D. Nix and T. W. Kenny, "What is the Young's modulus of silicon," J. Microelectromech. Syst., vol. 19, no. 2, pp. 229238, 2010.

[21] C. Prada, O. Balogun and T. W. Murray, "Laser-based ultrasonic generation and detection of zero-group velocity Lamb waves in thin plates," Appl. Phys. Lett., vol. 87, p. 194109, 2005.

[22] C. McKee, "Characterisation of Micromechanical Materials and MEMS Structures using Optically Generated Ultrasound," University of Strathclyde. PhD Thesis. 2014.
[23] B. Taitian, "Fitting refractive index data with the Sellmeier dispersion formula," Appl. Opt., vol. 23, no. 24, pp. 4477-4485, 1984.

[24] D. F. Edwards and E. Ochoa, "Infrared refractive index of silicon," Appl. Opt., vol. 19, no. 24, pp. 4130-4131, 1980.

[25] M. J. Weber, Ed., Handbook of Optical Materials, CRC Press, 1992.

[26] D. Alleyne and P. Cawley, "A two-dimensional Fourier transform method for the measurement of propagating multimode signals," J.Acoust. Soc. Am, vol. 89, no. 3, 1991.

[27] Fraunhofer Institute, [Online]. Available: http://www.ccl.fraunhofer.org/download/LA Wave.pdf.

[28] N. M. Jennett, G. Aldrich-Smith and A. S. Maxwell, "Validated measurement of Young's modulus, Poisson ratio, and thickness for thin coatings by combining instrumented nanoindentation and acoustical measurements," J. Mater. Res, vol. 19, no. 1, pp. 143 - 148, 2004

[29] C. B. Scruby, K. R. Jones and L. Antoniazzi, "Diffraction of elastic waves by defects in plates: Calculated arrival strengths for point force and thermoelastic sources of ultrasound.," J. Nondestruct. Eval, vol. 5, no. $3 / 4,1986$

Campbell McKee received the B.Sc. degree in applied physics and the Ph.D. degree in electrical and electronic engineering (specializing in using laser ultrasound to characterize MEMS structures and thin films) from the University of Strathclyde, Glasgow, U.K., in 2006 and 2014, respectively. $\mathrm{He}$ is now the Technical Manager at James Fisher Testing Services where his work involves the design and application of large scale structural monitoring solutions.

Brian Culshaw is professor emeritus at the University of Strathclyde, where he served as head of department, vice dean and research group leader. $\mathrm{He}$ is also a director of OptoSci limited. He has (too) many years' experience in the technology, realisation and application of fibre optic sensors and has also participated in conference organisation and professional society activities including acting as 2007 President of SPIE. After graduating with a Ph.D in electrical and electronic engineering (1970), he worked at University College London, Bell Northern Research Ottawa, and Stanford and Cornell Universities prior to joining Strathclyde in 1983.

Richard K. Leach received a B.S.C. degree in applied physics from Kingston University, Kingston, UK, in 1989, an M.S.C. degree in measurement systems from Brunel University, Uxbridge, UK, in 1994, a Ph.D. degree in surface metrology from University of Warwick, Coventry, UK, in 2000 and a D.S.C. degree in surface metrology from University of Warwick, Coventry, UK, in 2014. From 1990 to 2014, he was a Principal Research Scientist with the National Physical Laboratory, Teddington, UK.

$\mathrm{He}$ is currently professor in metrology at the University of Nottingham, Nottingham, UK. His research themes include the measurement of surface topography, development of methods for measuring 3D structures, development of methods for controlling large surfaces to high resolution in industrial applications and x-ray computed tomography.

$\mathrm{He}$ is a Fellow of the Institute of Physics, the Institution of Engineering \& Technology, the International Society of Nanomanufacturing, a Sustained Member of the American Society of Precision Engineering, a Chartered Engineer and a Chartered Physicist. $\mathrm{He}$ is a visiting professor at Loughborough University, UK and the Harbin Institute of Technology, China. 